\title{
Clinical Pharmacy and Pharmaceutical Care: Patient-Oriented Application of Pharmaceutical Expertise
}

\author{
Markus L. Lampert ${ }^{\star a}$, Stephan Krähenbühla ${ }^{a}$ Kurt E. Hersberger ${ }^{b}$, and Raymond G. Schlienger ${ }^{a}$
}

\begin{abstract}
Clinical Pharmacy is a health speciality describing the activities and services of the clinical pharmacist to develop and promote the rational and appropriate use of drugs, medicinal products, and devices by the individual as well as by the society.

Very closely related to the term of clinical pharmacy is the concept of pharmaceutical care. This means a new pharmacy practice paradigm which puts the patient into the centre. In the course of this practice, a pharmacist takes responsibility for a patient's drug-related needs with the aim of achieving definite outcomes that improve a patient's quality of life. At the University of Basel, clinical pharmacy and pharmaceutical care have developed constantly over the last twenty years. Examples from research and practice are given in this article, and areas for future work are outlined.
\end{abstract}

Keywords: Clinical pharmacy · Continuity of care · Drug safety · Drug-related problems · Pharmaceutical care · Pharmacotherapy

\section{Definitions of Clinical Pharmacy and Pharmaceutical Care}

Clinical Pharmacy is a commonly used term in pharmacy practice and in pharmacy literature, and goes back to the mid 1960s. As defined by the 'European Society of Clinical Pharmacy' (ESCP), it is a health speciality describing the activities and services of the clinical pharmacist to develop and promote the rational and appropriate use of drugs and medicinal products and devices by the individual as well as by the society [1]. Clinical pharmacy is concerned with the application of pharmaceutical ex-

\footnotetext{
${ }^{*}$ Correspondence: Dr. phil. nat. M.L. Lamperta

Tel.: + 41613286861

Fax: + 41612658864

E-Mail: lampertm@uhbs.ch

aDivision of Clinical Pharmacology \& Toxicology

University Hospital Basel

Hebelstrasse 2

$\mathrm{CH}-4031$ Basel

bInstitute of Clinical Pharmacy

Department of Pharmaceutical Sciences

Pharmacenter

University of Basel

Klingelbergstrasse 50

$\mathrm{CH}-4056$ Basel
}

pertise to help maximise drug efficacy and minimise drug related problems. However, the term 'Clinical Pharmacy' is often incorrectly used and interpreted. 'Clinical' does not necessarily mean an activity in a hospital setting. Clinical pharmacy includes all the services performed by pharmacists practising in hospitals, community pharmacies, nursing homes, home-based care services, clinics and any other setting where medicines are prescribed, dispensed or used. Clinical pharmacy is oriented to the analysis of population and individual needs of medicines, ways of administration, patterns of use and effects of drug therapy. The focus of attention moves from the drug to the single patient or population receiving drugs.

Clinical pharmacy is a relatively new discipline in the field of pharmacy which is based on the traditional 'columns' of the pharmaceutical sciences, i.e. pharmaceutical chemistry, biology, pharmaceutical technology and also pharmacology.

Very closely related to the term of clinical pharmacy is the concept of pharmaceutical care. In 1990, C.D. Hepler and L.M. Strand published the widely used definition of pharmaceutical care from a systems perspective. They state that pharmaceutical care is "the responsible provision of drug therapy for the purpose of achieving definite outcomes which improve a patient's quality of life" [2].
Thus, pharmaceutical care means a style of pharmacy practice provided for an individual patient and with the aim of improving the outcomes of therapy [3].

\section{Objectives of Clinical Pharmacy and Pharmaceutical Care}

The success of a pharmacotherapy is not only determined by the right choice of the active ingredient for a specific diagnosis, but by a number of important additional patient- and drug-related factors (Fig. 1). The way from the prescription of a drug to the desired effect on the patient's health is not straight forward [4].

Clinical pharmacy contributes to the rational and correct use of drugs in daily practice and searches for evidence for the best ways to do so. Clinical pharmacy focuses on the use of drugs in real life. For research purposes there are - in contrast to the classical pharmaceutical disciplines - only very limited possibilities to standardise environmental factors such as the patients' behaviour or concomitant drug treatment. Studies in this field cannot, therefore, find robust general rules like in the natural sciences, but concentrate on facts and interventions [15]. Relevant facts and factors influencing the therapeutic outcome of a drug therapy regimen are collated by specific and appropriate methods and techniques, and by 


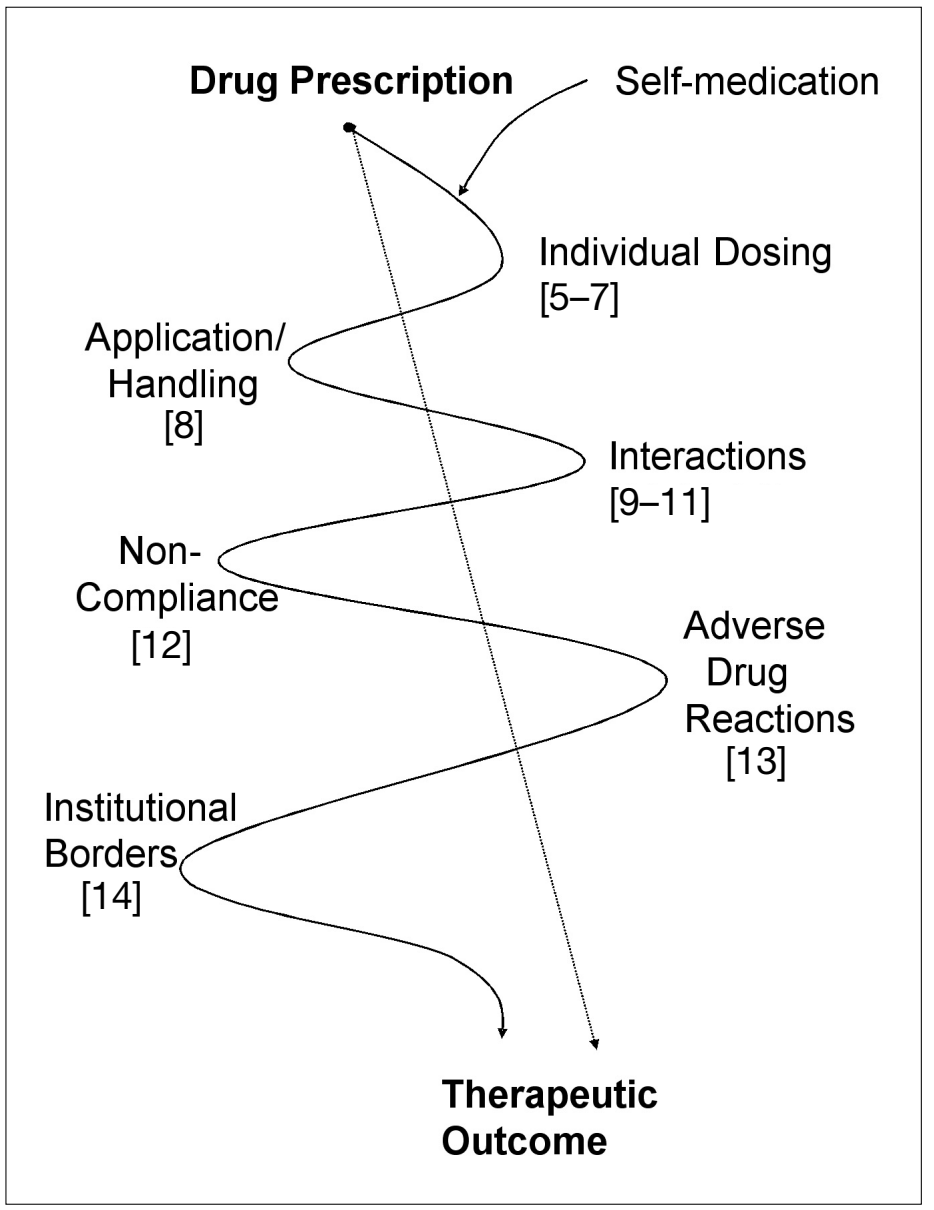

Fig. 1. Potential problems on the way to reach the optimal therapeutic outcome (adapted from [4]). Numbers in brackets refer to selected studies from the Institute of Clinical Pharmacy, University Basel.

doing so, these tools are constantly refined. Once these facts are described and assessed in enough detail, interventions are made and re-evaluated, and their impact (economic, clinical and humanistic, e.g. quality of life) is studied. Thus, research in clinical pharmacy and pharmaceutical care is positioned between the natural and the social sciences.

A simple example may illustrate the variety of problems which can arise during the drug therapy process: The treatment fails because the patient does not take his medicine. Behind this quite obvious problem, there may be several causes leading to completely different actions:

"The patient does not take the medicine because he/she...

- suffered adverse effects."

- cannot handle the drug, e.g. he/she is unable to swallow the tablet or to open the bottle."

- has not received enough information about his/her therapy."

- is not motivated to take it and does not see any benefit from it."

- he/she is not reimbursed for this drug from the health insurance company."

Clinical pharmacy in research intends to develop and evaluate tools and procedures for analysing problems and for finding solutions targeted to specified groups of patients or drugs. Pharmaceutical care in daily practice tries to analyse situations and to find an individual solution for a single patient.

\section{Clinical Pharmacy Activities}

First steps to assess and implement clinical pharmacists' activities at the University Hospital Basel in collaboration with the Department of Pharmacy were made in the late 1980s. At that time a local drug information centre run by pharmacists and clinical pharmacologists was set up at the Division of Clinical Pharmacology. Initially, the service was offered only to in-house medical and nursing staff [16]; later on, it was expanded to provide drug information to physicians and pharmacists outside the hospital [17][18].

Another important aspect of clinical pharmacy activities is therapeutic drug monitoring (TDM), which was introduced in the University Hospital Basel 25 years ago. TDM combines the measurement of drug concentrations in body fluids (especially in plasma, serum, whole blood, saliva) with pharmacokinetic and pharmacodynamic interpretations, taking the patient's individual situation into account. Hence, TDM can be a valuable and useful tool for some specific drugs to optimise and individualise pharmacotherapy. Furthermore, TDM may contribute to minimise the risk of concentration-dependent adverse drug reactions and therefore, may be an essential part of clinical management [19-22].

Many factors must play together to obtain a valid interpretation of a drug concentration measurement of which clinical consequences may be derived:

- A specific, accurate and precise analytical method must be available

- Timing of blood samples must be correct (e.g. drug distribution phase completed, sample for peak or trough level)

- Steady state concentrations must be reached

- Individual parameters of the patient have to be considered (e.g. renal function, plasma albumin concentration, etc.)

Several studies, however, demonstrate a high proportion of inappropriate drug level determinations [6][7], leading to unnecessary costs and potentially inadequate decisions based upon these drug levels. In this field, clinical pharmacy should help to develop interventions to bring pharmacokinetic and pharmacodynamic expertise directly to the patient's bed.

\section{Clinical Pharmacist at Ward Level}

The most recent development in clinical pharmacy at the University Hospital Basel led to the implementation of a clinical pharmacist at ward level. The aim of this position is to contribute to the optimisation of pharmacotherapy by direct interaction with the medical team (physicians and nurses) and the patient. Pharmaceutical expertise is thus available at the point of pharmacotherapeutic decision-making. To develop and implement future strategies in the treatment process, it is important to collect data on existing drug-related problems in the daily routine of a hospital setting. This data collection must be done in a structured manner. For this purpose, several classification systems for drug-related problems have been established and validated [23].

In the first few months since the implementation of a clinical pharmacy service on an internal medical ward with 77 beds, all pharmaceutical interventions were documented and classified using the PCNE Classification V5.00 [24]. This classification system allows an intervention to be described by four aspects: the problem itself, the probable cause, the intervention and the overall outcome. Figs 2 and 3 show the results of this data collection over a 20 -week period. These preliminary data demonstrate that drug choice, inappropriate dosing and 

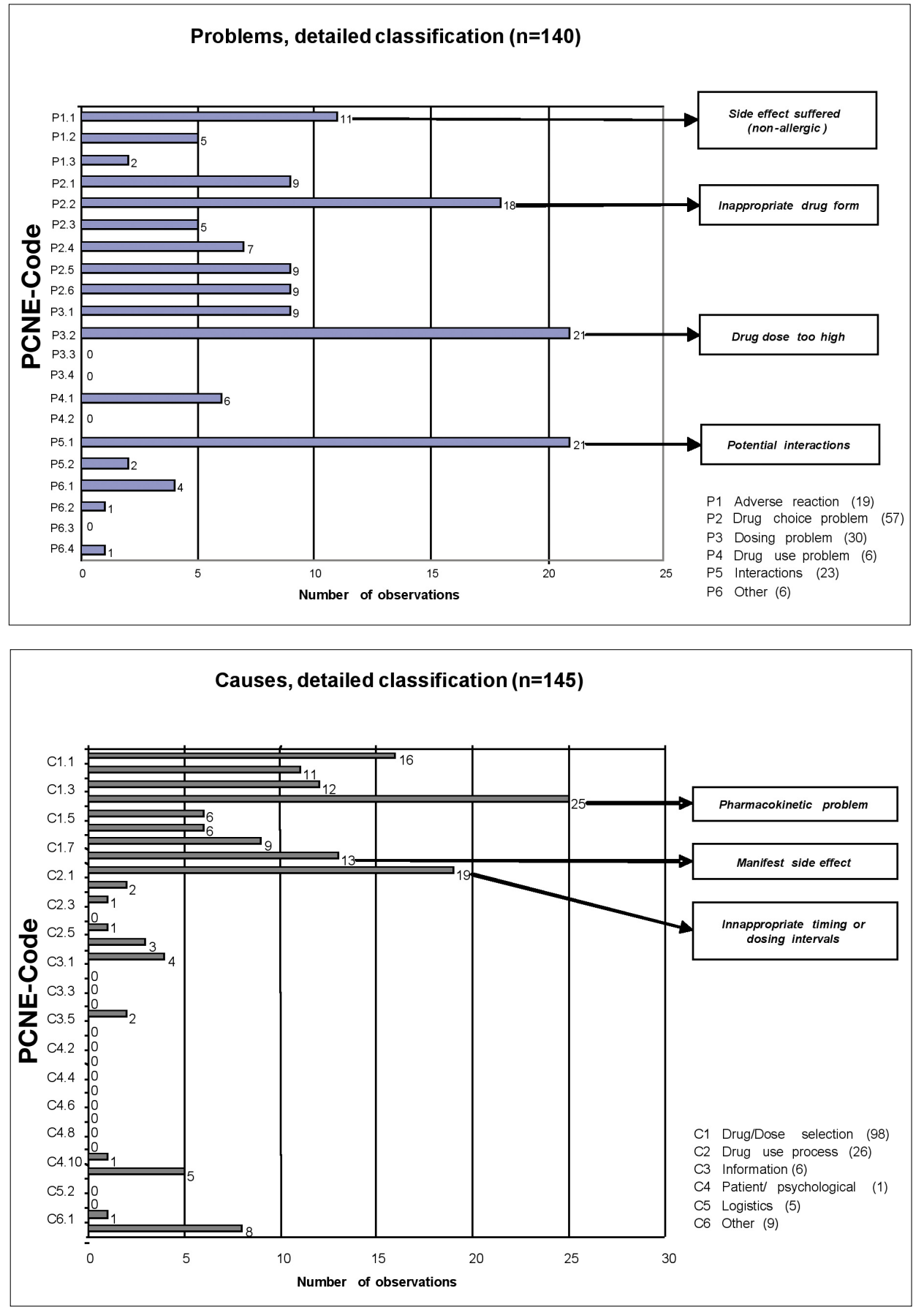

interactions are common problems in pharmacotherapy leading to suboptimal therapeutic outcomes. Clinical pharmacy at the point of decision, i.e. very close to the prescribing process, may detect such problems in a very early stage when they have had no negative impact yet. Detailed analysis of these data on drug-related problems can lead to the development of targeted interventions and to sustainable ameliorations in the drug use process.

\section{Future Challenges for Clinical Pharmacy in Research and Practice}

Drug treatment is becoming increasingly complex regarding the whole regimen of a patient and also regarding single drugs (e.g. proteins as drug substances which require specific knowledge about their handling and application). On the other hand, the length of hospital stay has decreased significantly over the past years. These two tendencies demand more intense care in the hospital and posthospital phase and require a high degree of self-management abilities from the patient. To assure these prerequisites for the optimal therapeutic outcome after a hospital stay, all the relevant information must be transferred between the different levels of care (Fig. 4). This continuity of care can be defined as:

"The degree to which the service system links episodes of treatment into a seamless, uninterrupted whole, in conformity with
Fig. 2. Classification of drug-related problems (according to PCNE Classification scheme V5.00) detected by a clinical pharmacist at ward level. Numbers in brackets show the total number of observations in the primary classification domains P1 to P6.
Fig. 3. Classification of causes of drug-related problems (according to PCNE Classification scheme V5.00) detected by a clinical pharmacist at ward level. Numbers in brackets show the total number of observations in the primary classification domains $\mathrm{C} 1$ to $\mathrm{C} 6$.

the needs of the patient. Continuity of care is a multidimensional concept including integration and co-ordination of services, communication among the various service providers and the stability of patient caregiver relationship over time" [25].

Whereas on the levels of daily activities and medical care continuity is more or less given, there is a gap on the level of pharmaceutical care. Until today, only in very few hospitals in Switzerland pharmacists are involved in bed-side patient care. At hospital discharge, the information from hospital to the community pharmacy is restricted to the discharge prescription. This information is not enough to support complex drug treatments after a hospital stay in order to contribute to an optimal compliance and to 


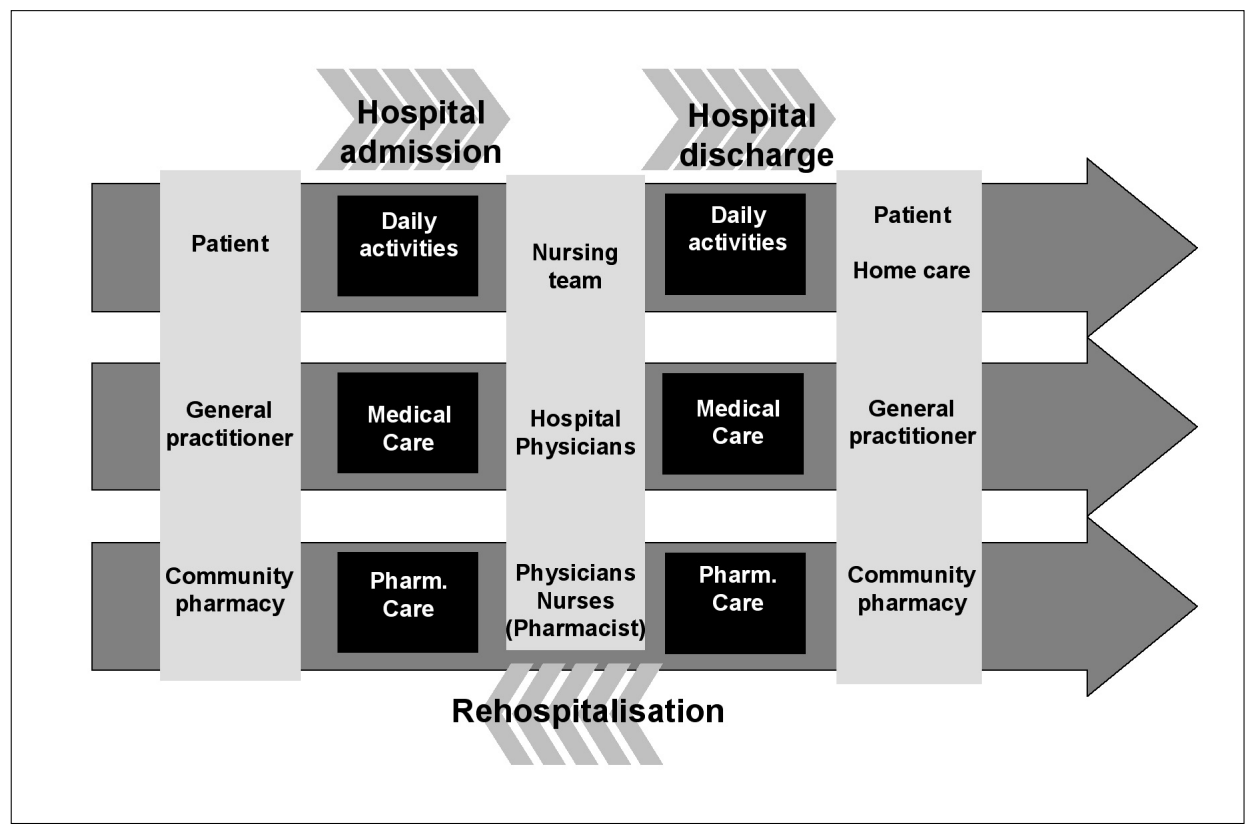

Fig. 4. Multidimensions of perihospital patient care (adapted from [14])

detect and prevent drug related problems. An important issue for the future development of clinical pharmacy will therefore be to find ways to bridge this gap in pharmaceutical care across institutional borders. The Institute of Clinical Pharmacy with its activities in both the hospital and the ambulatory setting, is well placed to focus future research on these issues of perihospital pharmaceutical care.

Received: December 29, 2005

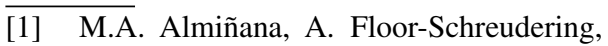
Y.A. Hekster, Y. Huon, G. Scroccaro, 'The need for Clinical Pharmacy', European Society of Clinical Pharmacy, http://www. associationhq.com/ESCP/pdf/Clinical_ Pharmacy.pdf (download 03-08-2005).

[2] C.D. Hepler, L.M. Strand, 'Opportunities and responsibilities in pharmaceutical care', Am. J. Hosp. Pharm. 1990, 47, 533.

[3] B.D. Franklin, J.W.F. van Mil, 'Defining clinical pharmacy and pharmaceutical care' (Editorial), Pharm. World Sci. 2005, 27, 137.

[4] W.E. Haefeli, Individualisierte Arzneimitteltherapie, Ther. Umsch. 2000, 57, 545.

[5] A.D. Falconnier, W.E. Haefeli, R.A. Schönenberger, C. Surber, M. Martin-Facklam, 'Drug dosage in patients with renal failure optimized by immediate concurrent feedback', J. Gen. Intern. Med. 2001, 16, 396.

[6] M.R. Mordasini, S. Krähenbühl, R.G. Schlienger, 'Appropriateness of digoxin level monitoring', Swiss Med. Weekly 2002, 132, 506.

[7] N. Affolter, S. Krähenbühl, R.G. Schlienger, 'Appropriateness of serum level determinations of antiepileptic drugs', Swiss Med. Weekly 2003, 133, 591.
[8] J. Kähni-Simonius, 'Arzneimittelinkompatibilitäten', Schweiz. Rundsch. Med. Prax. 1993, 82, 1320.

[9] S. Egger, J. Drewe, R.G. Schlienger, 'Potential drug-drug interactions in the medication of medical patients at hospital discharge', Eur. J. Clin. Pharmacol. 2003, 58, 773.

[10] B. Straubhaar, S. Krähenbühl, R.G. Schlienger, 'The prevalence of potential drugdrug interactions in patients with heart failure at hospital discharge', Drug Saf. $\mathbf{2 0 0 5}$, in press.

[11] A.E. Rätz Bravo, L. Tchambaz, A. Krähenbühl-Melcher, L. Hess, R.G. Schlienger, S. Krähenbühl, 'Prevalence of potentially severe drug-drug interactions in ambulatory patients with dyslipidaemia receiving HMG-CoA reductase inhibitor therapy', Drug Saf. 2005, 28, 263.

[12] I. Arnet, R.A. Schönenberger, R. Spiegel, W.E. Haefeli, 'Überzeugung als Fundament der Compliance und Techniken zur Compliance-Optimierung', Schweiz. Med. Wochenschr. 1999, 129, 1477.

[13] R.G. Schlienger, T.F. Lüscher, R.A. Schönenberger, W.E. Haefeli, 'Academic detailing improves identification and reporting of adverse drug events', Pharm. World. Sci. 1999, 21, 110.

[14] C. Wiesner, 'Arzneimittelsicherheit in der Perihospitalphase', Phil.-Nat.wiss. Dissertation, Universität Basel, 2001.

[15] M.P. Mobach, 'From the laboratory to pharmaceutical research', Pharm. World. Sci. 2001, 23(6), 205.

[16] F. Follath, C. Meier, E. Grimm, 'Die computergestützte Arzneimittelinformation', Schweiz. Med. Wochenschr. 1990, 120, 1845.

[17] C. Meier, 'Der Medikamenten-Informationsdienst im Spital', Schweiz. Rundsch. Med. Prax. 1993, 82, 1312.
[18] R.G. Schlienger, T.F. Lüscher, W.E. Haefeli, 'Clinical pharmacists' effect on drug information requests', Ann. Pharmacother. 1996, 30, 86.

[19] A.S. Gross, 'Best practice in therapeutic drug monitoring', Br. J. Clin. Pharmacol. 2001, 52, 5S.

[20] R. Spector, G.D. Park, G.F. Johnson, E.S. Vesell, 'Therapeutic drug monitoring', Clin. Pharmacol. Ther. 1988, 43, 345.

[21] J.K. Aronson, M. Hardman, 'ABC of monitoring drug therapy, Measuring plasma drug concentrations', $B M J$ 1992, 305, 1078.

[22] F. Abad-Santos, A.J. Carcas, C. Ibanez, J. Frias, 'Digoxin level and clinical manifestations as determinants in the diagnosis of digoxin toxicity', Ther. Drug. Monit. 2000, 22, 163.

[23] J.W. van Mil, L.O. Westerlund, K.E. Hersberger, M.A. Schaefer, 'Drug-related problem classification systems', Ann. Pharmacother. 2004, 38, 859.

[24] Pharmaceutical Care Network Europe, PCNE Classification for Drug Related Problems V5.00, www.pcne.org/dokumenter/PCNE\%20classification\%20V5.00. pdf (download 21-11-2005).

[25] O. Saarento, T. Oiesvold, S. Sytema, G. Gostas, M. Kastrup, O. Lonnerberg, S. Muus, M. Sandlund, L. Hansson, 'The Nordic Comparative Study on Sectorized Psychiatry, continuity of care related to characteristics of the psychiatric services and the patients', Soc. Psychiatry Psychiatr. Epidemiol. 1998, 33, 521. 